\title{
Study of Different Performance Parameters of Prototype Filter for Filter Bank Multicarrier Cognitive Radio
}

\author{
Er.A.S Kang ${ }^{1, *}$, Renu Vig ${ }^{2}$ \\ ${ }^{1}$ ECE Department, Panjab University Regional Centre, India \\ ${ }^{2}$ ECE Department, Panjab University, India
}

Copyright (C) 2015 Horizon Research Publishing All rights reserved

\begin{abstract}
Cognitive Radio has proven as an optimum technique for getting improved spectrum utilization by sharing the radio spectrum with licensed primary users opportunistically. The cognitive radio is a new paradigm to overcome the persisting problem of spectrum underutilization. Seeing the ever increasing demand of wireless applications, the radio spectrum is a valuable resource and in cognitive radio systems, trustworthy spectrum sensing techniques are required to avoid any harmful interference to the primary users. As cognitive radio possesses the capability to utilise the unused spectrum holes or white spaces so, there is a tremendous need to scan the large range of spectrum either for interference management or for primary receiver detection. Dynamic Spectrum Access techniques need to be implemented for the sake of better radio resource management and computational complexity analysis of multirate filter bank cognitive radio, where BER and $\mathrm{Eb} / \mathrm{No}$ are the performance metrics or governing parameters to affect the system performance using polyphase filter bank. The present paper deals with the study of effect of variation of number of subchannels $M$ at fix overlapping factor K of polyphase component of Filter Bank Multicarrier cognitive radio in terms of prototype filter length at $\mathrm{Lp}=\mathrm{K} * \mathrm{M}$.
\end{abstract}

Keywords FBMC, Cognitive-radio, Spectrum, Sub Channels, Filter Length

\section{Introduction}

The recent interest in cognitive radio based research has attracted a great deal of attention in spectrum sensing and detection of radio users in the environment. The primary objective is to maximize the probability of detection without losing much on the probability of false alarm while minimizing the complexity and time to sense and detect the radio. The limited spectrum for dense wireless communications and inefficient spectrum utilization necessitates a new communication paradigm cognitive radio which can exploit the unutilized spectrum opportunistically. The evolving research efforts are in the field of cognitive radios covering the different Physical Layer Aspects such as cognitive process, different modulation techniques which are utilised for the signal transmission in CR and varying spectrum sensing techniques.CR as a future wireless communication system is characterized as a system, which is able to adapt its transmission and reception parameters on the basis of cognitive interaction with the wireless environment in which it actually operates. There are several reasons that make spectrum sensing, a practically challenging task. A very low required SNR for primary user detection, multi-path fading and time dispersion effects of wireless channels which tend to complicate the spectrum sensing problem and the frequently changing noise level with time and location causes the noise power uncertainity a big issue of concern for primary user detection [1].

\section{Physical Layer of Cognitive Radio}

Spectrum sensing is the main task of the PHY layer of a cognitive radio. Cognitive radio reflects the situations where both primary and secondary users occupy the same channel space as in licensed band situations. It is also responsible for spectrum sensing and reconfiguration of the transmission parameters. Cognitive Radio can reconfigure its operating frequency, modulation, channel coding and output power without hardware replacement, this is the most significant difference between cognitive radio network and other wireless networks physical layer [2-4]. Software defined radio (SDR) based RF front-end transmitters and receivers are required for configurability of cognitive radio networks. Implementing RF front-end, heavy-weight signal processing algorithms, detecting weak signals, presence of PU while there are secondary users, are significant sensing problems in Cognitive Radio [1]. 


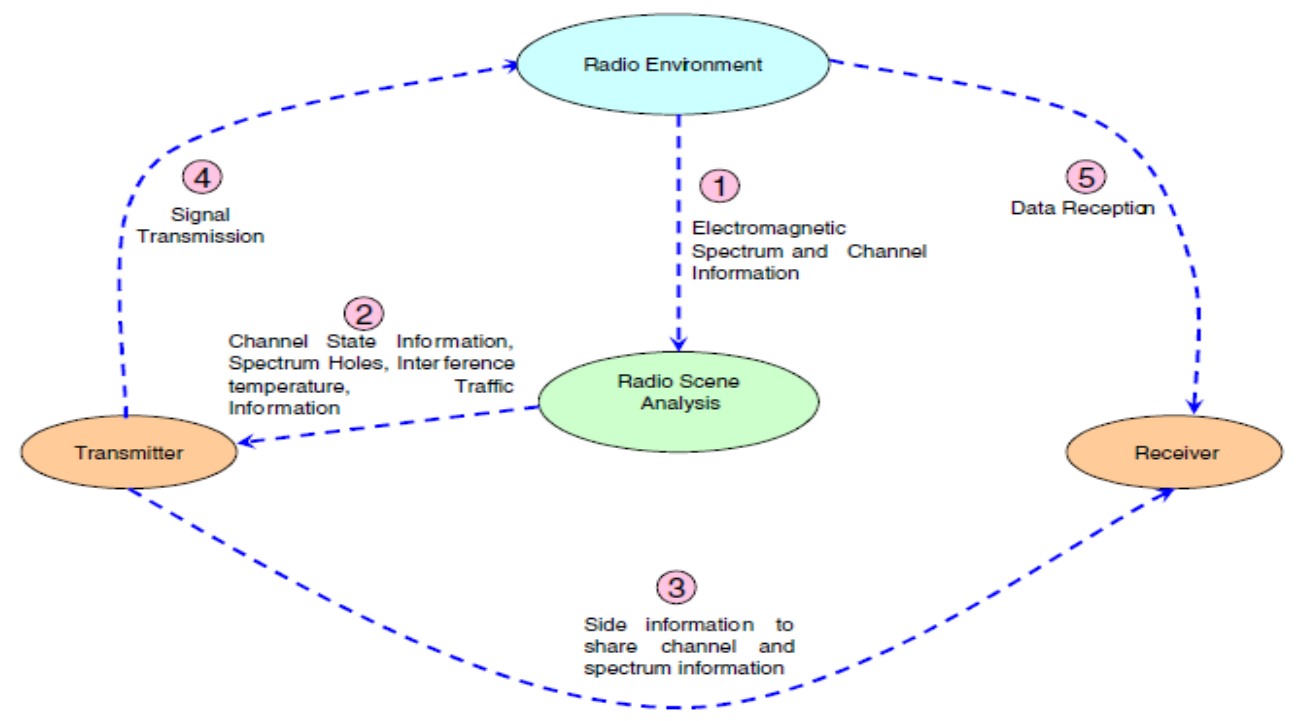

Figure 1. CR Block Diagram of OSI Model

\subsection{Main Features of Physical Layer}

PHY Transport: 802.22 use Orthogonal Frequency Division Multiplexing (OFDM) as transport mechanism. Modulation: QPSK, 16-QAM and 64-QAM are supported. Coding: Convolutional Code is Mandatory. Turbo, LDPC or Shortened Block Turbo Codes are optional but recommended. Pilot Pattern: Each OFDM / OFDMA symbol is divided into sub-channels of 28 sub-carriers of which 4 are pilots, which are inserted every 7 sub-carriers. No frequency domain interpolation is required. Net Spectral Efficiency: $0.624 \mathrm{bits} / \mathrm{s} / \mathrm{Hz}-3.12 \mathrm{bits} / \mathrm{s} / \mathrm{Hz}$. Spectral Mask: 802.22 have adopted the Spectral Mask requirements proposed by FCC.(200 tap FIR filter required). Fig.1 shows the Block diagram of CR-OSI Model showing the importance of Physical layer.

\section{Earlier Related Work}

Sheryl Ball et al [2005] has focussed on the potential of $\mathrm{CR}$ in military and emergency service uses. Some of the issues involved in adapting cognitive radio technology consumer markets were examined and some potential advantages to $\mathrm{CR}$ defined networks for consumers were highlighted with some possible service designs and pricing systems[2].Tero Ihalainen et al[2006] introduced a new low complexity per-subcarrier channel equalizer for FBMC transceiver for high-rate wideband communication over doubly-dispersive channel and analyzed its performance. It was shown that the coded error-rate performance of FBMC is somewhat better than that of the OFDM reference[3].Qiwei Zhang et al[2007] discussed that benefits of $\mathrm{CR}$ are clear when used in emergency situations. The idea of applying CR to emergency network is to alleviate this spectrum shortage problem by dynamically accessing free spectrum resources.CR is able to work in different frequency bands and various wireless channels and supports multimedia services such as voice, data and video. Reconfigurable radio architecture is proposed to enable the evolution from the traditional Software Defined Radio(SDR) to CR[4].Shuang Liu [2008]investigated the motive, definition and paradigms of cognitive radio. The author described the system model used comprising of one primary user and one secondary user with perfect channel side information available at both its transmitter and receiver and channel fading gains[5].Xin Kang et al[2008] studied the optimal power control policies for fading channels in cognitive radio networks considering both the transmit and interference power constraints. For each of the constraints peak power and average power are investigated. The author derived the optimal power allocation strategies in terms of maximizing the ergodic capacity of the secondary user when channel state information is available to the transmitter and the receiver[6].Musbah Shaat et al [2009] addressed the problem of resource allocation in multicarrier based CR networks. The objective is to maximize the downlink capacity of the network under both total power and interference introduced to the primary users constraints. The optimal solution has high computational complexity, which makes it unsuitable for practical applications and hence a low complexity suboptimal solution is proposed. The performance of using FBMC instead of OFDM in CR systems is investigated. Simulation results show that the proposed resource allocation algorithm with low computational complexity achieves near optimal performance and proves the efficiency of using FBMC in CR context[7].Qihang Peng et al[2010] discussed the analysis and simulation of sensing deception in fading cognitive radio networks [8].M Bahadir Celebi et al[2010] proposed cognitive radio implementation by using standard wireless communication laboratory equipments such as signal generator and spectrum analyzer. Equipment's are controlled through MATLAB instrument control toolbox to carry out 
CR capabilities specified by IEEE 802.22 WRAN standard. The aim of the work is to provide a CR environment for spectrum sensing algorithms to perform a comparative study considering wireless microphone signals for research and educational purposes[9].Shixian Wang et al [2011] discussed the cognitive radio simulation environment realization based on autonomic communication [10].Ajay $\mathrm{Kr}$ Sharma et al [2011] analysed the BER performance of Cognitive Radio Physical Layer over Rayleigh channel under different channel encoding schemes, digital modulation schemes and channel conditions. It has been anticipated from simulation study that the performance of the communication system degrades with the increase of noise power [11].Musbah Shaat and Fauzi Bader [2012] considered a multicarrier based CR network. The goal is to maximize the total sum rate of CR system while ensuring that no excessive interference is induced to the primary system [12].Satwant Kaur [2013] studied the wireless networks with cognitive radio technology and discussed its various functions and capabilities as a new networking paradigm for future wireless communication [13].

\section{Problem Formulation}

Large parts of assigned spectrum are underutilized while the increasing number of wireless multimedia applications lead to spectrum scarcity. Cognitive radio is an option to utilize non used parts of the spectrum that actually are assigned to primary services. The benefits of cognitive radio are clear in the emergency situations. Current emergency services rely much on the public networks. This is not reliable in public networks where the public networks get overloaded. The major limitation of emergency network needs a lot of radio resources. The idea of applying Cognitive Radio to the emergency network is to alleviate this spectrum shortage problem by dynamically accessing free spectrum resources. Cognitive Radio is able to work in different frequency bands and various wireless channels and supports multimedia services such as voice, data and video. The literature survey shows that the performance of FBMC based $\mathrm{CR}$ system can be enhanced under different radio environment. There is a need to work out on the following gaps that have been affecting the performance of cognitive radio.

[a] The spectral efficiency of OFDM based Cognitive Radio is less as compared to FBMC based CR due to the insertion of Cyclic Prefix in OFDM results in high spectral leakage in its prototype filter. FBMC is severely affected by non linear distortions but the resulting out of channel leakage is still lower compared to OFDM. Hence, non linear distortion must be minimized for efficient FBMC systems.

[b] The problem of vertical and horizontal sharing of radio spectrum and transmission power control exists.

[c] The problem of Interference in radio environment under different nodes for primary and secondary user locations results in spectrum underutilization. [d] Optimization and tradeoff of power, capacity constraints variables under $\mathrm{CR}$ radio environment should be considered.

[e] The Resource Allocation Strategies to combat inherent interference on primary user are less efficient.

[f] The performance of CR in terms of Energy Spectrogram and Power Spectrogram under different modulation and coding techniques in different radio environment (fading channels) without picocell, microcell, femtocell approach with different indoor and outdoor propagation models using spectrum sensing approach is not effective.

[g] Lack of Interlayer Optimization (Cross Layer design) results in degradation in radio resource management.

[h] The problem of Congestion in ISM bands adversely affecting the quality of communication persist in Conventional OFDM based network without Dynamic Spectrum Access which is not there in CR based network (with dynamic spectrum access).

The present study puts its focus on Performance Enhancement of Filter Bank Multicarrier (FBMC) based Cognitive Radio (CR) in adaptive, opportunistic, autonomic domain under different strategic conditions of wireless environment. By introducing techniques to improve the spectral efficiency and minimizing the spectrum underutilization, hence improving the overall performance of FBMC based CR.

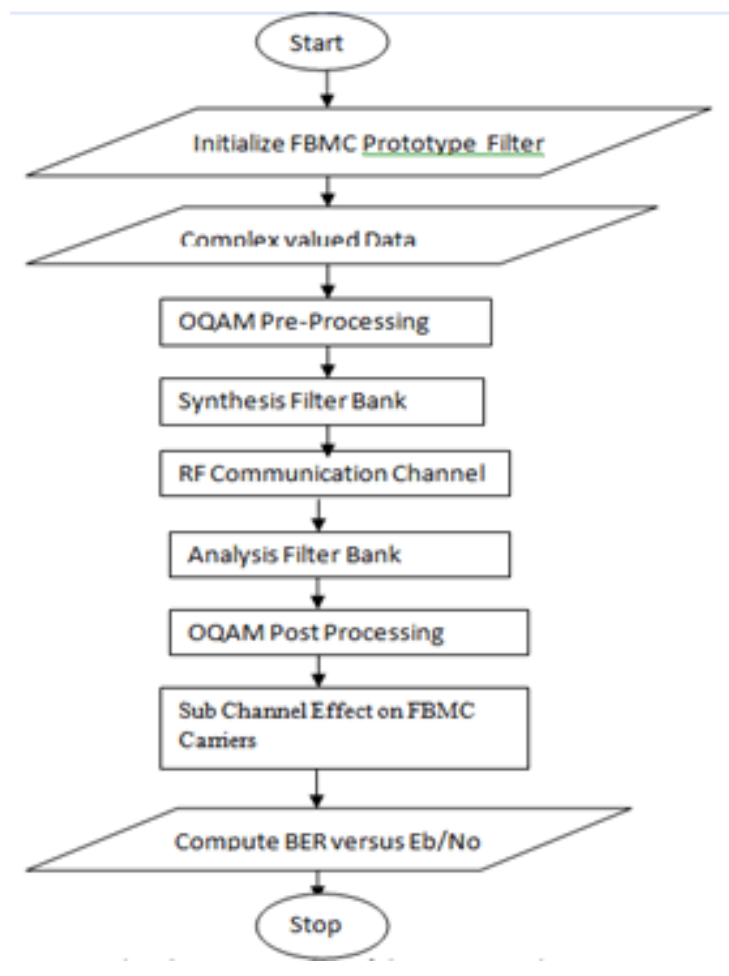

Figure 2. Flowchart for the process flow

\section{Flowchart for Present Study}


A flowchart has been prepared for study of effect of sub channels on prototype filter length for fbmc cognitive radio. The following system parameters have been used in the present investigation. K-Overlapping Factor, M-Number of Subchannels, $\mathrm{D}=$ Channel Delay, $\mathrm{L} p=$ Prototype Filter Length. The distinctive feature of the fbmc design technique has ability to provide improved frequency selectivity through the use of longer and spectrally well shaped prototype filters. In the present case, more emphasis has been laid on the $\mathrm{L} p=\mathrm{KM}-\mathrm{D}$ as a specific prototype filter length, under the assumption of Delay D as zero.

\section{Results and Discussions}

Different Graphic plots have been obtained between the various parameters namely Number of Subchannels M,Bit Error Rate BER, Signal to Noise Ratio SNR, Prototype Filter Length $\mathrm{Lp}=\mathrm{K}^{*} \mathrm{M}$ at a fix value of Overlapping factor $\mathrm{K}=4$. The spectrum sensing is performed by measuring the signal strength at the outputs of the subcarrier channels at the receiver. The Cognitive Radio system is able to transmit over the direct link more than that when the direct link is blocked for all subcarriers in the source side. The impact of the present study of FBMC CR is highlighted through the role of number of subchannels. Readjustment of various parameter levels leads to optimization between different radio environment parameters under varying strategic conditions. The computational complexity of the FBMC cognitive radio is studied under the effect of K,M and Lp. For FBMC system, the prototype coefficients are assumed to be equal to PHYDAS coefficients with overlapping factor $\mathrm{K}=4$. Actually, the entire process here involves the three steps. fixing the subcarriers, matching the subcarriers and re-adjusting the assigned subcarriers as per the system requirement on an average basis. For optimization and tradeoff sake, the number of subcarriers is taken to be greater than 8.The literature survey on FBMC shows that the different subcarriers are adjusted in such a way that the interference to the primary user by secondary users is kept to a minimum. Moreover, the impact of different constraints values on the system performance is investigated. This paper describes a linear assignment problem to select and match some subcarriers for transmission and use the rest only for direct transmission. The FBMC CR in physical layer is a potential candidate for future wireless communication system. A Bandwidth of $10 \mathrm{Mhz}$ with $\mathrm{M}=64,128,256,512$ subcarriers have been taken into consideration in the present scenario. The subcarriers are allocated sequentially to the users with optimum results.Figure3. shows BER versus SNR plot at different values of M.BER is found to decrease with increase at different values of SNR. The trend for $M$ values has been clearly depicted here. At $\mathrm{M}=64, \mathrm{BER}=1.45$ at $\mathrm{SNR}=0$ $\mathrm{db} . \mathrm{BER}=1.1 \quad$ at $\quad \mathrm{SNR}=1 \mathrm{db} . \mathrm{BER}=0.75 \quad$ at $\mathrm{SNR}=2 \mathrm{db} . \mathrm{BER}=0.45$ at $\mathrm{SNR}=3 \mathrm{db} . \mathrm{BER}=0.3 \quad$ at $\mathrm{SNR}=4 \mathrm{db} . \mathrm{BER}=0.1$ at $\mathrm{SNR}=5 \mathrm{db}$.It is very clear that at higher values of M,initial values of BER are found to be higher than 1.45 at $\mathrm{SNR}=0 \mathrm{db}$. The values of $\mathrm{SNR}$ chosen are well within the range $(-5 \mathrm{db}$ to $+30 \mathrm{db})$ as per specifications of IEEE802.22 Standard for FBMC Cognitive Radio.Fig.4.shows the matlab plot between Lp and BER which shows that beyond $\mathrm{Lp}=500, \mathrm{BER}$ is found to rise to a value $10^{-0.2}$ till $\mathrm{Lp}=2000$. Fig. 5 shows matlab plot between $\mathrm{Lp}$ and SNR which clearly shows SNR decrease beyond $\mathrm{L} p=10^{3}$. Fig. 6 shows matlab plot between $\mathrm{M}$ and BER which indicates that beyond $\mathrm{M}=10^{2}$, BER increases to $10^{-0.2}$.Fig.7 shows with $\mathrm{M}$ more than $10^{2}$, Lp becomes more than $10^{3}$.In fig.8,SNR decreases to $2 \mathrm{db}$ beyond $\mathrm{M}=10^{2}$.Various performance parameters have depicted that with increasing $\mathrm{BER}, \mathrm{SNR}$ is found to decrease and vice versa.

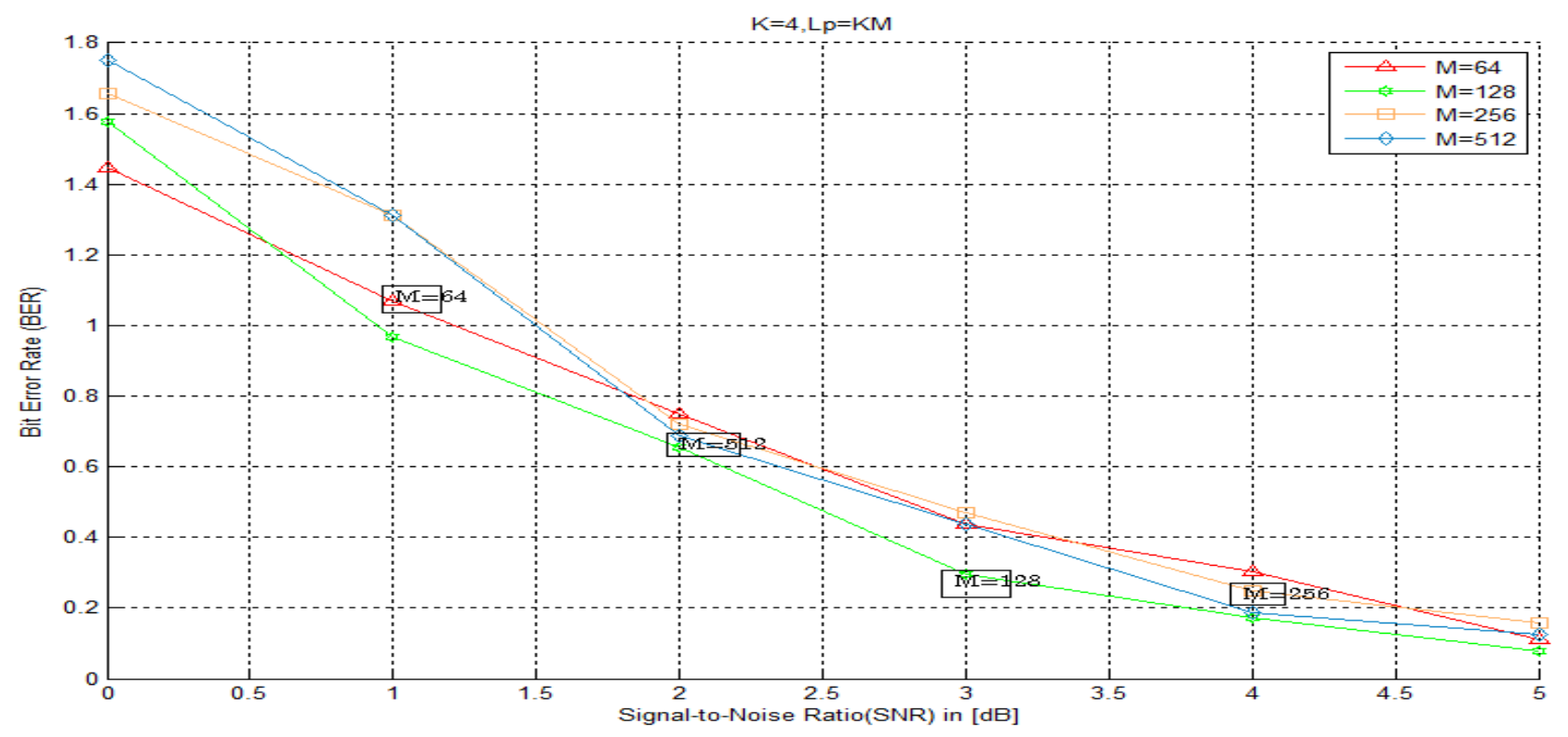

Figure 3. Matlab plot between $\mathrm{BER}$ and $\mathrm{SNR}$ at $\mathrm{K}=4, \mathrm{Lp}=\mathrm{KM}$ with $\mathrm{M}=64,128,256,512$ 


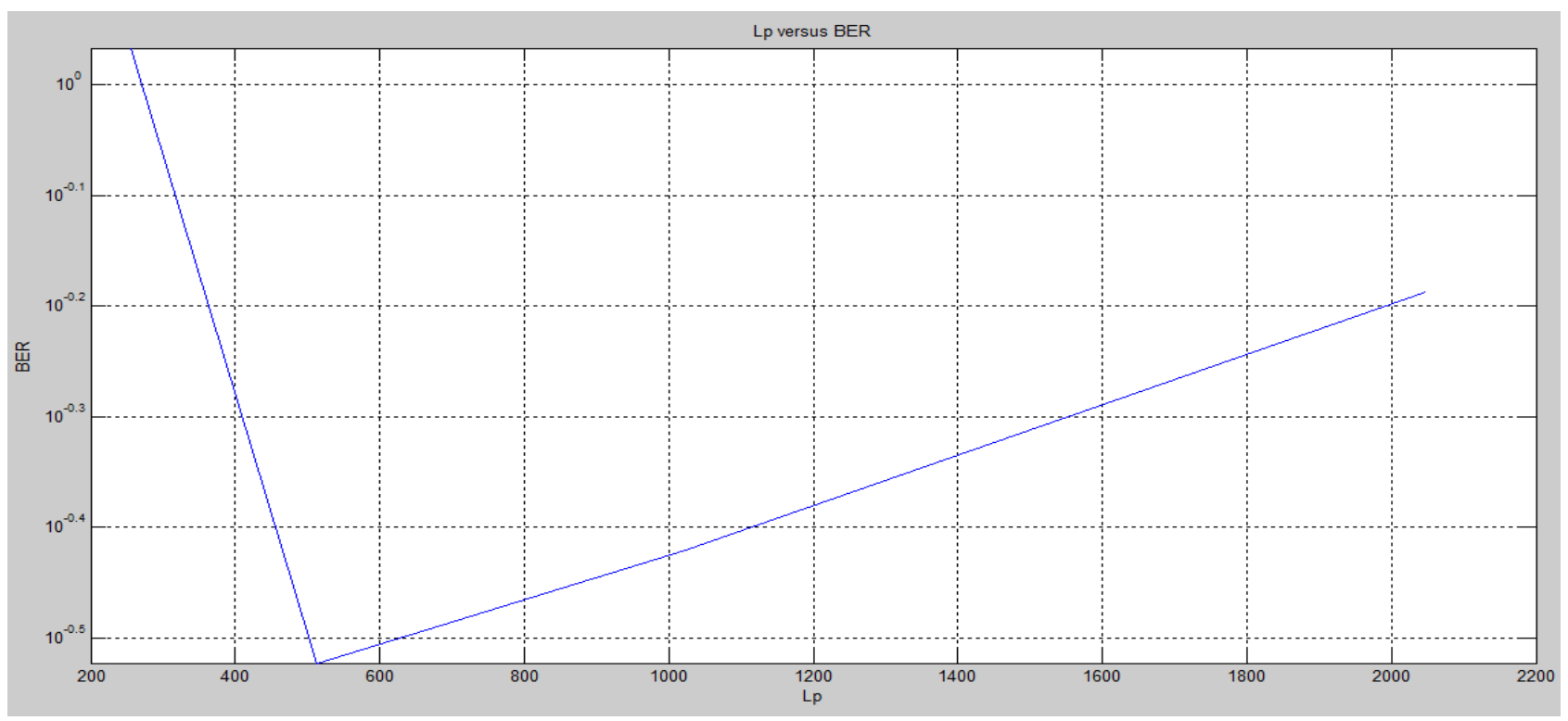

Figure 4. Matlab plot between Lp and BER

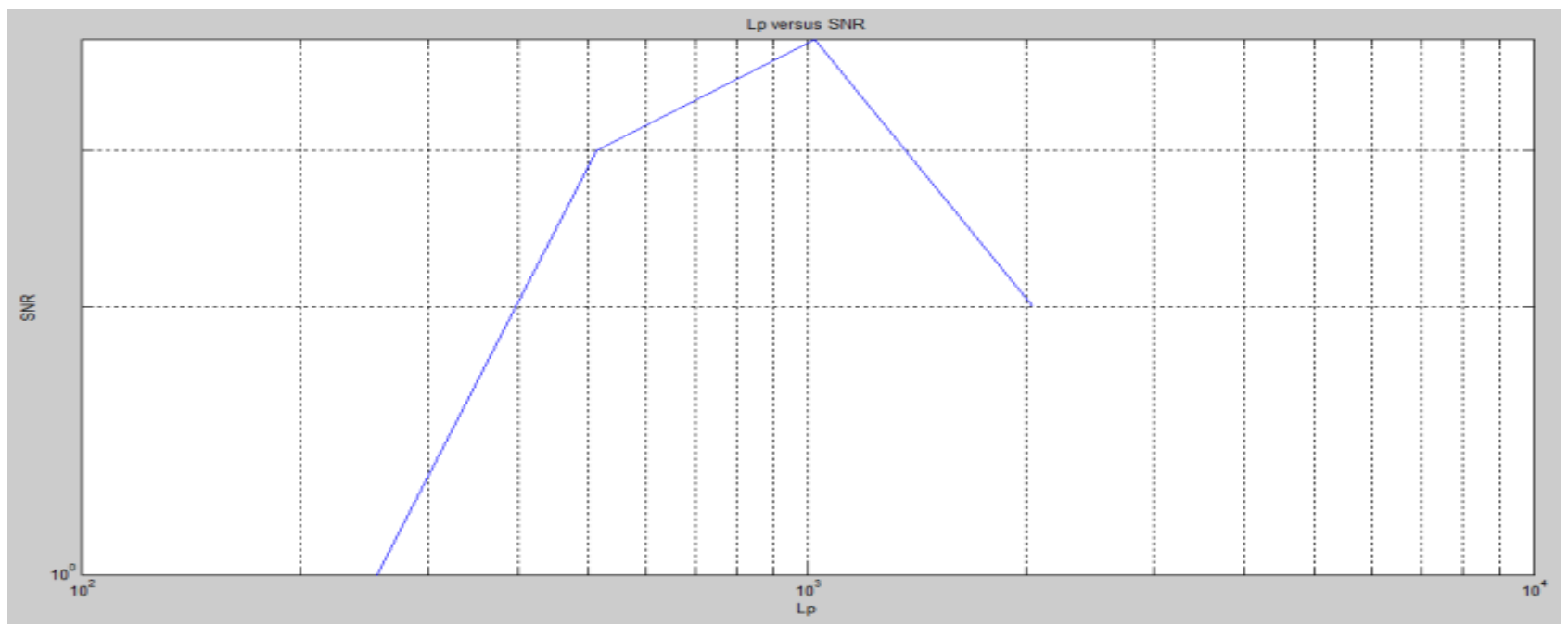

Figure 5. Matlab plot between Lp and SNR

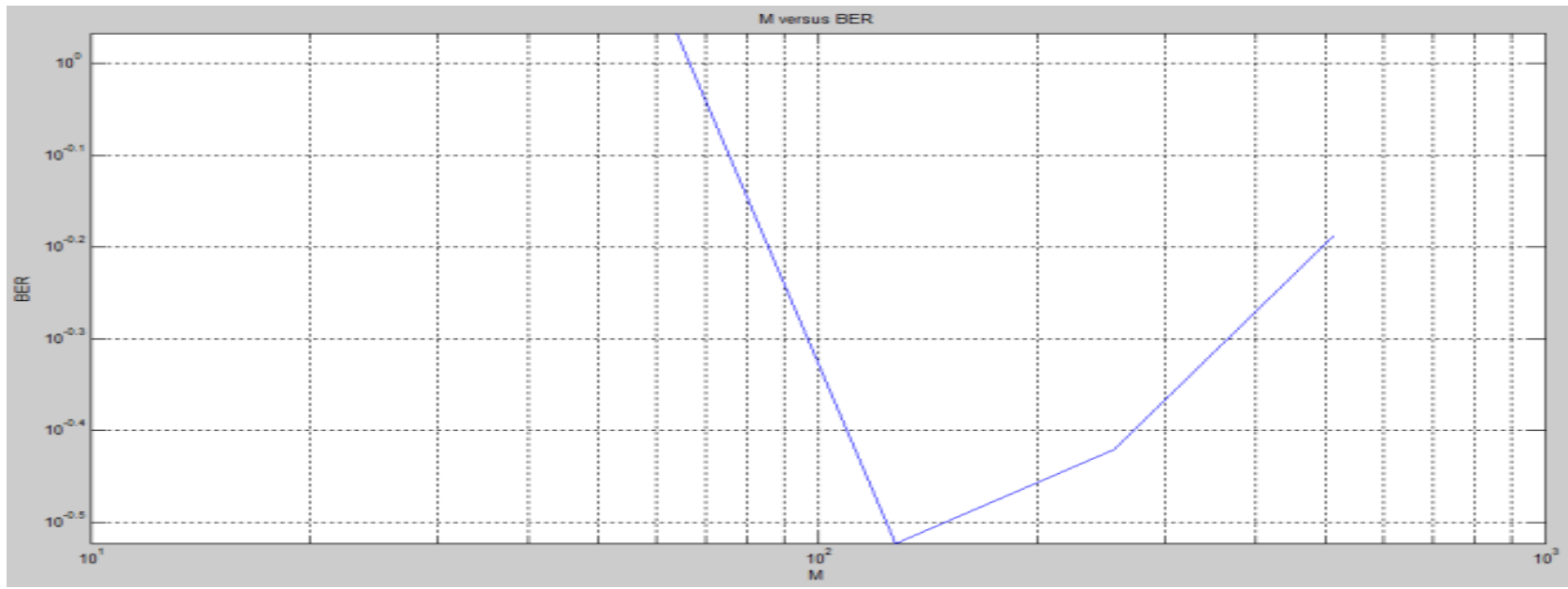

Figure 6. Matlab plot between M and BER 


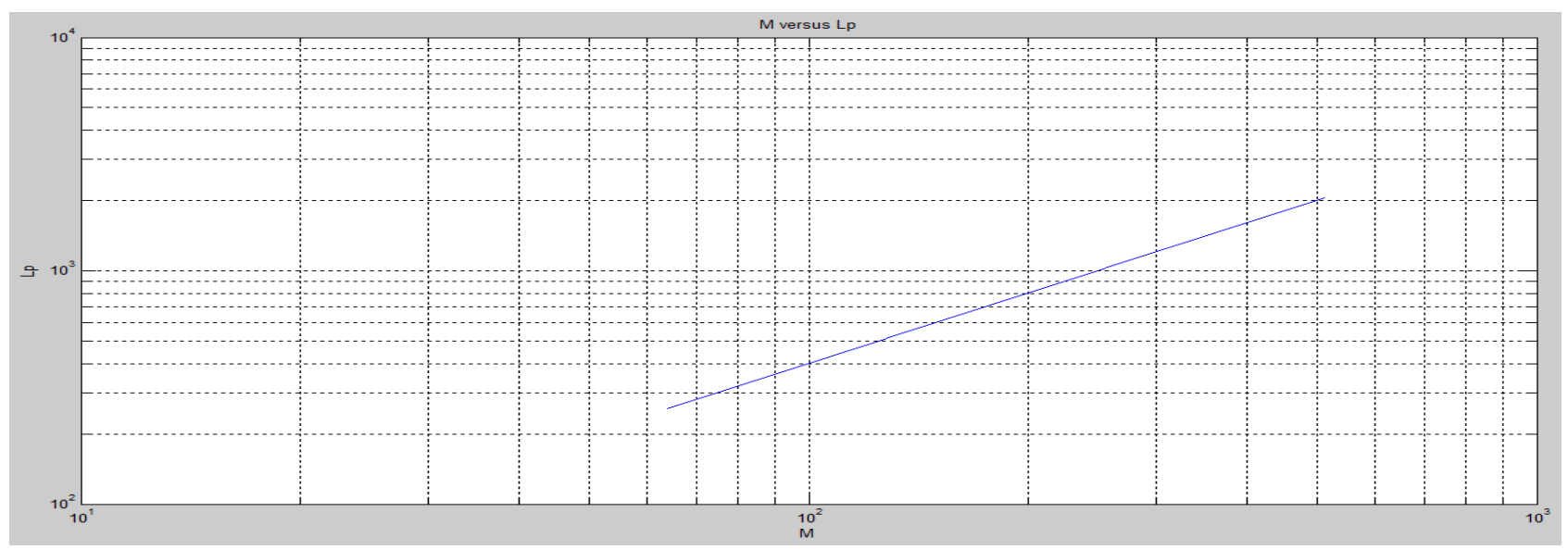

Figure 7 Matlab plot between M and Lp

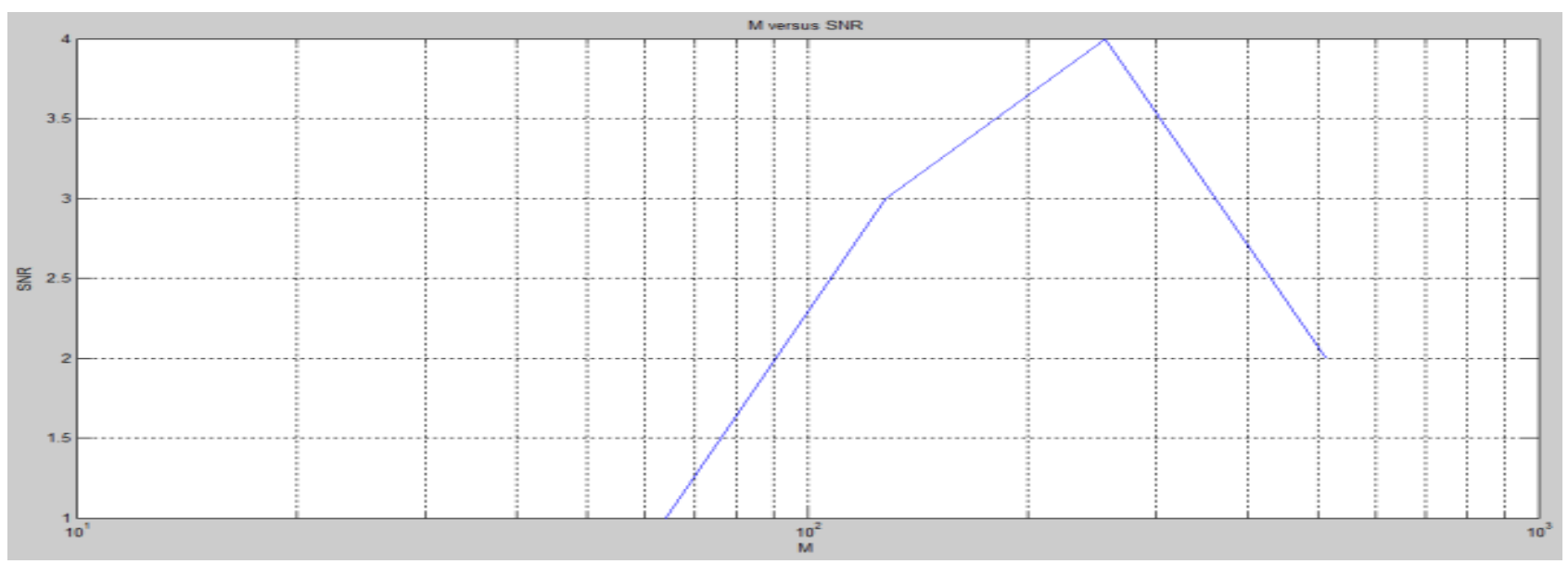

Figure 8. Matlab plot between M and SNR

\section{Conclusions}

Cognitive radio networks have a promising future and have excellent applications of wireless networks. The signal processing prospectives of CR have significant impact in $\mathrm{CR}$ technology in its performance enhancement. The present paper shows the comparative analysis of number of subchannels $\mathrm{M}$ for $\mathrm{FBMC}$ prototype filter length at $\mathrm{Lp}=\mathrm{K}^{*} \mathrm{M}$ using BER and $\mathrm{Eb} / \mathrm{No}$ as performance measuring indicators. Analytical results reveal that the tough requirements on the probability of detection of primary users can only be met with enhancement of channel capacity and spectral efficiency through the variation of $\mathrm{Lp}, \mathrm{K}$ and $\mathrm{M}$ factor corresponding to the design and implementation of modified FBMC prototype filter under different strategic conditions or ubiquitous environment. It is quite apparent that the prototype filter modified version must have the essential characteristics like linear phase with unity roll off to minimize delay and circuit complexity and cost. Moreover, the modified prototype filter should have the transmission zeros at the frequencies which are integral multiples of subchannel spacing, for independent and accurate channel measurements. Attenuation increasing with frequency, to offer the increasing level of protection from/to primary users in cognitive radio is another requirement that is mandatory. So, with these constraints, the main flexibility parameters in the system design are number of subchannels, overlapping factor, which determines the total number of filter coefficients, and the option for partial filter bank design, to equip a low rate user in uplink in an efficient manner. The preliminary results discussed in this paper clearly show that FBMC is sensitive to the RF impairments. The effect of impairments on FBMC cognitive radio can be studied further using analysis and synthesis filter banks with the aid of unified signal models. Comparative analytical and simulation study of different prototype filter lengths namely, $\mathrm{Lp}=\mathrm{KM} . \mathrm{KM}-1, \mathrm{KM}+1$ can be easily extended to the future version.

\section{Impact of Study}

The expected outcome of proposed research will be helpful in detecting interference at primary receiver and speed and reliability of detection in Cognitive cycle of CR system. The Spread Spectrum Detection in terms of Power and with wide frequency range hidden in the noise can be done. The hidden node problem due to the multipath fading in the propagation between primary transmitter and sensing 
receiver can be studied by applying learning and intelligence approach. The most of the CR will have to autonomously work in multiservice, multi technology and multiuser environment and it has to adapt the different parameters under different conditions. Vertical and Horizontal sharing of radio spectrum will be possible to some extent with efficient spectrum space opportunities, spectrum mobility and transmission power control. The CR must be capable of spectrum sensing and operating over wide radio spectrum range, emulate many radio technologies and different modulation schemes which cause various hardware challenges. The present study will be useful for optimum selection of hardware components which will minimize circuit complexity and cost and less chances of interference. OFDM based CR network performance will improve with the application of multirate signal processing in multicarrier wireless communication. Better Radio Resource Management is possible. The problem of Congestion in ISM bands which adversely affects the quality of communication will be reduced by CR networks based on Dynamic Spectrum Access.

\section{Future Directions}

As Cognitive radio technology is an important innovation for the future of communications and likely to be a part of the new wireless standards, becoming almost a necessity for situations with large traffic and interoperability concerns. Moreover CR is devised to be used with telecommunications or computer network related disciplines but there are inadequate facilities to provide robustness and effective security. To overcome this, existing technologies will increase the complexity and new types of attack are possible. Therefore, innovative ideas are required to provide security to Cognitive Radio Networks and make them robust against crucial attacks, especially the attacks inherent to the Cognitive Radio functionality. So to make Cognitive Radio systems trustworthy, dependable and efficient, a comprehensive energy efficient mechanism is required to identify, remove or mitigate the attacks at any phases of the Cognitive Cycle. The study has its impact on the design and development of cognitive radio system using Filter Bank Multicarrier Approach[14-22].

\section{REFERENCES}

[1] J. Mitola and G. Q. Maguire, "Cognitive Radios: Making Software Radios More Personal", IEEE Personal. Communication., Vol. 6, no. 4, pp. 13-18, August 1999.

[2] Sheryl Ball et al., "Consumer Applications of Cognitive Radio Defined Networks,”Proc.IEEE 2005,pp.518-525.

[3] Tero Ihalainen, T. H. Stitz et al.,"Channel Equalization in Filter Bank based Multi carrirer Modulation For Wireless Communications", Hindawi Publishing Corporation,
EURASIP Journal on Advances in Signal Processing, Vol. 2007,pp.1-18,2007.

[4] Qiwei Zhang et al, "Towards Cognitive Radio for Emergency Networks,”Chapter1,pp.1-26.

[5] Shuang Liu,"Capacity Comparison for Fading Channels under Average and Peak Interference Power Constraints in Underlay Cognitive RadioSystem,"Proc.Stanford.edu,pp.1-8 ,2008.

[6] Xin Kang ,Y C Liang et al.,'Optimal Power Allocation for Fading Channels in Cognitive Radio Networks under Transmit and Interference Power Constraints," Proc. IEEE International Conference on Communications ICC2008,pp.3568-3572,2008.

[7] Musbah Shaat et al., "Computationally Efficient Power Allocation Algorithm in Multicarrier- Based Cognitive Radio Networks, OFDM and FBMC systems", EURASIP Journal on Advances in Signal Processing,Vol.2010,pp.1-13,2010.

[8] Qihang Peng, Pamela C Cosman et al., "Analysis and Simulation of Sensing Deception in Fading Cognitive Radio Networks",Proc.2010 IEEE,pp.234-242,2010.

[9] M. Bahadur Celebi, Huseyin Arslan et al.,"Spectrum Sensing Testbed Design for Cognitive Radio Applications,"Proc. International Conference on Cognitive Radio Applications,2010,pp.1-4.

[10] Shixian Wang, Hengzhu Liu et al.,"Cognitive Radio Simulation Environment Realization based on Autonomic Communication,"Proc.2011IEEEThird International Conference on Communication Software and Networks ICCSN,pp.402-407,2011.

[11] Ajay Kr Sharma et al.,"BER Performance Analysis of Cognitive Radio Physical Layer over Rayleigh fading channel, "International Journal of Computer Applications, Vol 25-no.11,pp.25-29,July 2011.

[12] Musbah Shaat and Fauzi Bader, "Comparison of OFDM and FBMC Performance in Multi-Relay Cognitive Radio Network,"Proc.2012 International Symposium on Wireless Communication Systems,pp.756-760,2012.

[13] Satwant Kaur,"Intelligent Wireless Network -Cognitive Networks, IETE Technical Review,Vol.30,Issue 1,Jan-Feb2013,pp.6-12.

[14] A.S.Kang and Renu Vig, "Analysis of Effect of Variable Number of Subchannels on the Performance of Filter Bank Multicarrier Prototype Filter,"Journal of Electrical \& Electronic Systems, Kang and Vig, J Electr Electron Syst 2014,3:1 pp.1-7.

[15] A.S.Kang and Renu Vig, "BER Performance Analysis of Filter Bank Multicarrier using Sub band Processing for Physical Layer Cognitive Radio" Journal of Electr Electron Syst 2014, 3:3.

[16] A.S.Kang and Renu Vig, "Performance Analysis of Filter Bank Multicarrier Cognitive Radio for Physical Layer under Binary Symmetric Radio Fading Channel,'International Journal of Computer Applications(0975-8887),Vol.93(6),May2014.pp.27-32.

[17] A.S.Kang and Renu Vig, "Computational Complexity Analysis of FBMC-OQAM under Different Strategic Conditions,"Proc.2014 RAECS UIET Panjab University 
Chandigarh,06-08March,2014.978-1-4799-2291-8/14/\$31.00 Copyright 2014IEEE.pp.1-9.

[18] A.S.Kang and Renu Vig, "Study of Filter Bank Multicarrier Cognitive Radio under Wireless Fading Channel,"Proc.IEEE -IACC International Conference 2014,978-1-4799-2572-8/1 4/\$31.00Copyright2014IEEE,pp.209-214.

[19] A.S.Kang et al "Trade-off Between AND and OR Detection Method for Cooperative Sensing in Cognitive Radio," Proc.IEEE-IACC International Conference 2014,978-1-4799 -2572-8/14/\$31.00Copyright2014IEEE,pp.209-214.pp.395-3 99.
[20] A.S.Kang,Jaisukh Paul Singh et al, "Cognitive Radio:State of Research Domain in Next Generation Wireless Networks-A Critical Analysis,"International Journal of Computer Applications(0975-8887)Vol.74-No.10,July2013,pp.1-9.

[21] A.S.Kang,Rajwinder Singh et al, "Cognitive Radio New Dimension in Wireless Communication-State of Art,"IJCA, Vol.74-No.10,July 2013,pp.10-19.

[22] JaisukhPaulSingh,A.S.Kang et al "Cooperative Sensing for Cognitive Radio:A Powerful Access Method for Shadowing Environment,SPRINGER-Journal of Wireless Personal Communications,2014.Journal:11277WIRE Article No..2088,Pgs15 